Short Communication

\title{
International Society for Extracellular Vesicles and International Society for Cell and Gene Therapy statement on extracellular vesicles from mesenchymal stromal cells and other cells: considerations for potential therapeutic agents to suppress coronavirus disease-19
}

\section{Verena Börger $^{1, \dagger}$, Daniel J. Weiss ${ }^{2, \dagger}$, Johnathon D. Anderson ${ }^{3}$, Francesc E. Borràs ${ }^{4}$,}

Benedetta Bussolati ${ }^{5}$, David R.F. Carter ${ }^{6}$, Massimo Dominici ${ }^{7}$, Juan M. Falcón-Pérez ${ }^{8,9,10}$, Mario Gimona ${ }^{11}$, Andrew F. Hill ${ }^{12}$, Andrew M. Hoffman ${ }^{13}$, Dominique de Kleijn ${ }^{14}$, Bruce L. Levine ${ }^{15}$, Rebecca Lim ${ }^{16}$, Jan Lötvall ${ }^{17}$, S. Alex Mitsialis ${ }^{18}$, Marta Monguió-Tortajada ${ }^{19}$, Maurizio Muraca ${ }^{20}$, Rienk Nieuwland ${ }^{21}$, Anna Nowocin ${ }^{22}$, Lorraine O'Driscoll ${ }^{23}$, Luis A. Ortiz ${ }^{24}$, Donald G Phinney ${ }^{25}$, Ilona Reischl ${ }^{26}$, Eva Rohde ${ }^{27,28}$, Ralf Sanzenbacher ${ }^{29}$, Clotilde Théry ${ }^{30}$, Wei Seong Toh ${ }^{31}$, Kenneth W. Witwer ${ }^{32,33, *}$, Sai Kiang Lim $^{34, *}$, Bernd Giebel ${ }^{1, *}$

${ }^{1}$ Institute for Transfusion Medicine, University Hospital Essen, University of Duisburg-Essen, Essen, Germany

${ }^{2}$ Department of Medicine, University of Vermont, Burlington, Vermont, USA

${ }^{3}$ Department of Otolaryngology, Stem Cell Program, University of California, Davis, Davis, California, USA

${ }^{4}$ REMAR-IVECAT Group, Health Science Research Institute Germans Trias i Pujol (IGTP), Can Ruti Campus, and Nephrology Service, Germans Trias i Pujol University Hospital, Badalona, Spain

${ }^{5}$ Department of Molecular Biotechnology and Health Sciences, University of Torino, Torino, Italy

${ }^{6}$ Department of Biological and Medical Sciences, Oxford Brookes University, Oxford, UK

${ }^{7}$ Department of Medical and Surgical Sciences of Children and Adults, University Hospital of Modena, Modena, Italy

${ }^{8}$ Exosomes Laboratory, Center for Cooperative Research in Biosciences (CIC bioGUNE), Basque Research and Technology Alliance (BRTA), Derio, Spain

${ }^{9}$ Centro de Investigación Biomédica en Red de enfermedades hepáticas y digestivas (CIBERehd), Instituto de Salud Carlos III, Madrid, Spain

${ }^{10}$ IKERBASQUE, Basque Foundation for Science, Bilbao, Spain

${ }^{11}$ GMP Unit and EV-TT Transfer Center, Spinal Cord Injury \& Tissue Regeneration Center Salzburg (SCI-TReCS), Paracelsus Medical University (PMU), Salzburg, Austria

${ }^{12}$ La Trobe Institute for Molecular Science, La Trobe University, Bundoora, Australia

${ }^{13}$ School of Veterinary Medicine, University of Pennsylvania, Philadelphia, Pennsylvania, USA

${ }^{14}$ Department of Vascular Surgery, University Medical Center Utrecht and Netherlands Heart Institute, Utrecht, the Netherlands

${ }^{15}$ Center for Cellular Immunotherapies at the Perelman School of Medicine, University of Pennsylvania, Philadelphia, Pennsylvania, USA

${ }^{16}$ Department of Obstetrics and Gynaecology, Hudson Institute of Medical Research, Monash University and The Ritchie Centre, Melbourne, Australia

${ }^{17}$ Krefting Research Centre, Institute of Medicine, Sahlgrenska Academy at University of Gothenburg, Gothenburg, Sweden

${ }^{18}$ Department of Pediatrics, Harvard Medical School and Boston Children's Hospital, Boston, Massachusetts, USA

${ }^{19}$ ICREC Research Program, Health Science Research Institute Germans Trias i Pujol (IGTP), Can Ruti Campus, and Cardiology Service, Germans Trias i Pujol

University Hospital, Badalona, Spain

${ }^{20}$ Department of Women's and Children's Health, University of Padua, Padua, Italy

${ }^{21}$ Laboratory of Experimental Clinical Chemistry, Department of Clinical Chemistry and Vesicle Observation Center, Amsterdam UMC, Location AMC, University of

Amsterdam, Amsterdam, the Netherlands

${ }^{22}$ Biotherapeutics, National Institute for Biological Standards and Control (NIBSC), Medicines and Healthcare Products Regulatory Agency, Hertfordshire, UK

${ }^{23}$ School of Pharmacy and Pharmaceutical Sciences and Trinity Biomedical Sciences Institute, Trinity College Dublin, Dublin, Ireland

${ }^{24}$ Department of Environmental and Occupational Health, University of Pittsburgh, Pittsburgh, Pennsylvania, USA

${ }^{25}$ Department of Molecular Therapeutics, The Scripps Research Institute, Jupiter, Florida, USA

${ }^{26}$ Federal Office for Safety in Health Care (BASG) and Austrian Agency for Health and Food Safety (AGES), Institute Surveillance, Vienna, Austria

${ }^{27}$ Department of Transfusion Medicine, University Hospital, Salzburger Landeskliniken GesmbH (SALK), Salzburg, Austria

${ }^{28}$ GMP Unit, Spinal Cord Injury \& Tissue Regeneration Centre Salzburg (SCI-TReCS), Paracelsus Medical University (PMU), Salzburg, Austria

${ }^{29}$ Section Tissue Engineering and Cell Therapeutics, Paul-Ehrlich-Institut, Federal Institute for Vaccines and Biomedicines, Langen, Germany

${ }^{30}$ Institut Curie/INSERM U932/PSL Research University, Paris, France

${ }^{31}$ Faculty of Dentistry, National University of Singapore, Singapore

${ }^{32}$ Department of Molecular and Comparative Pathobiology, The Johns Hopkins University School of Medicine, Baltimore, Maryland, USA

${ }^{33}$ Department of Neurology, The Johns Hopkins University School of Medicine, Baltimore, Maryland, USA

${ }^{34}$ Institute of Molecular and Cellular Biology, Agency for Science, Technology and Research, Singapore

\footnotetext{
* Correspondence: Bernd Giebel, PhD, Institute for Transfusion Medicine, University Hospital Essen, University of Duisburg-Essen, Virchowstr. 179, 45147 Essen, Germany.

* Kenneth W. Witwer, Johns Hopkins University School of Medicine, 733 N. Broadway, MRB 827, Baltimore, MD 21205, USA

* Sai Kiang Lim, Institute of Molecular and Cellular Biology, Agency for Science, Technology and Research, Singapore

E-mail addresses: kwitwer1@jhmi.edu (K.W.Witwer), Lim_Sai_Kiang@imcb.a-star.edu.sg (S.K. Lim), bernd.giebel@uk-essen.de (B. Giebel).
} 
A R T I C L E I N F O

Article History:

Received 1 May 2020

Accepted 2 May 2020
STATEMENT: The International Society for Cellular and Gene Therapies (ISCT) and the International Society for Extracellular Vesicles (ISEV) recognize the potential of extracellular vesicles (EVs, including exosomes) from mesenchymal stromal cells (MSCs) and possibly other cell sources as treatments for COVID-19. Research and trials in this area are encouraged. However, ISEV and ISCT do not currently endorse the use of EVs or exosomes for any purpose in COVID-19, including but not limited to reducing cytokine storm, exerting regenerative effects or delivering drugs, pending the generation of appropriate manufacturing and quality control provisions, pre-clinical safety and efficacy data, rational clinical trial design and proper regulatory oversight. (c) 2020 International Society for Cell and Gene Therapy. This is an open access article under the CC BY-NC-ND license. (http://creativecommons.org/licenses/by-nc-nd/4.0/)
First described in December 2019, the severe acute respiratory syndrome associated with coronavirus disease-19 (COVID-19) quickly evolved into a pandemic, with severe and increasing worldwide morbidity and mortality. Although most infected patients have mild to moderate symptoms or are even asymptomatic, older patients and those with pre-existing chronic diseases are at greater risk of developing serious complications, such as pneumonia or multiple organ failure. COVID-19 respiratory infection is marked by dysregulated immune responses leading to significant respiratory pathology as well as increased probabilities for multi-organ pathologies. While the inflammatory pathways are still being elucidated, notable components include increased circulating levels of proinflammatory cytokines and other mediators, including interleukin-6 (IL-6), interleukin-1 $\beta$ (IL-1 $\beta$ ), induced protein 10 (IP10) and monocyte chemoattractant protein-1 (MCP-1) $[4,6,41]$. There are also significant alterations in circulating inflammatory cell populations, with initial lymphocytosis followed by severe lymphopenia, with increased ratios of helper to regulatory T cells $[4,6,30]$. Since dysregulated immune responses and the cytokine storm are triggers for development of acute respiratory distress syndrome, an increasing effort and current clinical trials are focused on immune therapeutic approaches, such as IL-1 blockade (anakinra), IL-6 receptor blockade (tocilizumab) and Janus kinase inhibition [22]. In parallel, there are a rapidly increasing number of cell-based therapy investigations, mostly utilizing mesenchymal stromal cells (MSCs) [12]. These are based on supporting pre-clinical data for use of MSCs delivered either systemically or intratracheally in pre-clinical models of acute lung injuries and on demonstration of safety of systemic MSC administration in recent trials for acute respiratory distress syndrome resulting from other etiologies [15, 21].

Among the cell-based therapy investigations for COVID-19, some registered clinical trials aim to utilize extracellular vesicles (EVs) prepared from MSC-conditioned media rather than the cells themselves. MSC-EVs will be administered intravenously (ChiCTR2000030484) or by inhalation (NCT04276987, ChiCTR2000030261). The rationale for these approaches is based on a relatively small but growing number of investigations in pre-clinical lung injury and sepsis models in which MSC-EV preparations were described as being as safe and effective as-if not more than-their parent cells $[19,40]$. The approach is further supported by a growing body of literature on the therapeutic potential and mechanisms of EVs in a wide range of diseases, including recent positive results in a steroidrefractory graft-versus-host disease patient treated with MSC-EVs and in a single-center, randomized, placebo-controlled phase 2/3 clinical pilot study on chronic kidney disease patients treated with MSC-EVs [14, 25].

The mechanisms by which EVs exert their beneficial effects, as well as their site(s) of action, remain incompletely understood. Nonetheless, effects observed in a range of pre-clinical non-COVID-19 model systems suggest that they may also have efficacy against COVID-19. For example, systemic administration of MSC-EV preparations modulated immune responses such as elevated cytokine storms in relevant lung disease models, including acute lung injury and sepsis $[17,20,23,24,27,35,38,42]$. Notably, in Escherichia coli-induced pneumonia mouse models, MSC-EV administration was found to enhance phagocytosis of bacteria [9, 23]. In a pig model, MSC-EVs were shown to attenuate influenza virus-induced acute lung injury, among other conditions, by inhibiting influenza virus replication [11]. Disease-attenuating effects on inflammatory immune responses following MSC-EV administration have also been observed in other disease models [2]. In an ischemic stroke model, for example, syste mic MSC-EV administration reduced stroke-induced lymphopenia and proinflammatory immune responses in the brain and periphery, resulting in overall improvement of disease symptoms [7, 37]. These preliminary observations support MSC-EV administration as a potential treatment option for COVID-19.

However, the specific scientific rationale for the administration of MSC-EV and other EV treatments in COVID-19 patients needs to be better understood and justified. For example, MSC-EVs do not necessarily suppress immune responses, but rather modulate them. Specifically, they seem to moderate acute immune responses toward regulatory responses, with the latter inducing tolerance and restoring homeostasis [43-45]. While tolerance induction in graft-versus-host disease and other non-infectious diseases may be beneficial, it might also have severe adverse effects in the presence of replicating pathogens. Although influenza and E. coli infections were attenuated in selected models $[9,11,23]$, other viruses and bacteria might conceivably expand in an uncontrolled manner in induced tolerogenic environments.

A number of additional issues should be considered before administering MSC-EVs to COVID-19 patients. These include the source of MSCEVs. MSCs are a heterogeneous cell entity that can be obtained from different tissues. Even if derived from the same tissues, they may display interindividual and possibly clone-specific functional differences [28, 29, 31, 36]. Indeed, side-by-side comparison of four MSC-EV preparations harvested from the conditioned media of different donor-derived bone marrow MSCs demonstrated significant variations in cytokine content [14]. Whether this correlates with therapeutic potency is not yet clear; however, in the example of the ischemic stroke model, it was demonstrated that MSC-EV preparations with comparable particle and protein contents can significantly differ in potency. While some preparations effectively suppressed stroke symptoms, others failed to exert therapeutic activities [37]. Furthermore, in an acute lung injury model, EVs from young, but not aged, MSCs alleviated lipopolysaccharideinduced acute lung injury [10].

Potentially, heterogeneity of EV potency due to different sources, preparations, aging and other factors could be resolved by generating immortalized clonal MSC lines that could be rigorously tested for EV production and potency [5]. Still, apart from their immunomodulatory capabilities, MSC-EVs apparently also control additional biological processes, some with approved therapeutic functions [1] and

$\dagger$ These authors contributed equally to this work. 
others that might trigger unforeseen side effects. Just recently, it was found that adipose-derived MSC-EVs had higher thrombogenic activity than bone marrow-derived MSC-EVs [3, 34]. Thus, the source of parental cells might increase thrombosis risk. Coupled with the finding that activation of complement pathways and an associated procoagulant state seem to result in catastrophic microvascular injury syndrome in a proportion of severe COVID-19 cases [18], MSC-EV administration could even be counterproductive in COVID-19.

To this end, it is imperative that stringent "identity" and "potency" parameters are defined and potential side effects addressed before MSC-EV or other EV preparations are released for therapeutic applications [16, 32, 39]. To date, many groups use inhouse MSC-EV manufacturing and characterization strategies, mainly for pre-clinical studies [2]. Protocols fulfilling Good Manufacturing Practice (GMP) criteria are sparse, and just a few have been published $[8,26,33]$. For product candidates, studies focusing on safety and clinical pharmacology need to be performed. Results of such studies are mandatory to provide guidance for adjustment of manufacturing, storage, dosing and administration of EV-based therapeutics in specific target diseases.

We would like to refer to a recent statement by ISCT on the use of MSCs in COVID-19 [13] and one by the Italian STEMnet ${ }^{1}$, as many of the same considerations apply to MSC-EVs or other EVs. Governmental organizations, health care providers and clinical investigators must take the lead by insisting that clinical uses of EVs follow appropriate scientific, regulatory and ethical guidelines and are approved only after a rigorous review by duly empowered agencies. The ethical guidelines produced by the World Health Organization are a useful baseline ${ }^{2}$. The urgency of the current outbreak does not justify administration of EVs in uncontrolled compassionate use settings and does not obviate the need to register clinical trials, obtain informed consent from patients or proxies and otherwise comply with good clinical practice. In particular, even limited compassionate use should employ wellcharacterized MSC-EV preparations produced through strict GMP conditions under the oversight of the relevant national regulatory entity. Additional outbreak-specific measures may be needed, including establishing simplified clinical protocols for hospitalized patients, such as the World Health Organization COVID-19 core protocol; minimizing risks to trial integrity ${ }^{3}$; and changing logistics of trial participant visits (e.g., implementation of remote assessments) as well as protocol changes for the sake of hazard minimization, which may need to be implemented and reported, in Europe, to the Institute for Research in Biomedicine Barcelona after the fact. Certainly, to foster developments, it is helpful to have regulatory flexibility and support from sources such as the US Food and Drug Administration special emergency program for possible therapies, the Coronavirus Treatment Acceleration Program $^{4}$, the European Medicines Agency (EMA) COVID-19 Pandemic Task Force ${ }^{5}$, the EMA guidance for medicine developers and companies on COVID- $19^{6}$ and the guidelines for clinical trials published by an EMA-coordinated group ${ }^{7}$ or the Medicines and Healthcare Products Regulatory Agency ${ }^{8}$, respectively. Most or all of the considerations covered for cell-based therapies are also applicable to EV investigations.
In conclusion, to mitigate the risk of potentially life-threatening side effects, ISCT and ISEV strongly urge that the potential benefits and risks in the use of MSC-EVs for COVID-19 be weighed carefully against available pre-clinical data in relevant animal models and clinical data from relevant MSC clinical trials and that any use of EVs be carefully evaluated through rational clinical trial design, employing well-characterized EV preparations produced under strict GMP conditions and under the proper regulatory oversight.

\section{Funding}

No funding was received.

\section{Declaration of competing interest}

JDA is the co-founder of an exosome therapeutics company called Somos Therapeutics, Inc. BG is a scientific advisory board member with Evox Therapeutics and Innovex Therapeutics SL. MG has a consulting and advisory role with MDimune. BLL has stock and other ownership interests with Tmunity Therapeutics; has received honoraria from Novartis, Terumo and AstraZeneca; and has a consulting or advisory role with Brammer Bio/ThermoFisher Viral Vector Services, Avectas, Immuneel, Ori Biotech and Vycellix. SKL is the founder of Paracrine Therapeutics and has a scientific advisory role with Ilias Biologics and ExoCo. SAM is the inventor of intellectual property licensed by $\mathrm{BCH}$ to United Therapeutics Corp.

\section{Author contributions}

Drafting the manuscript: VB, DJW, KWW, SKL, BG. All authors have approved the final article.

\section{References}

[1] Arslan F, Lai RC, Smeets MB, Akeroyd L, Choo A, Aguor EN, Timmers L, van Rijen HV, Doevendans PA, Pasterkamp G, et al. Mesenchymal stem cell-derived exosomes increase ATP levels, decrease oxidative stress and activate PI3K/Akt pathway to enhance myocardial viability and prevent adverse remodeling after myocardial ischemia/reperfusion injury. Stem Cell Res 2013;10:301-12.

[2] Börger V, Bremer M, Ferrer-Tur R, Gockeln L, Stambouli O, Becic A, Giebel B. Mesenchymal stem/stromal cell-derived extracellular vesicles and their potential as novel immunomodulatory therapeutic agents. Int J Mol Sci 2017;18.

[3] Chance TC, Rathbone CR, Kamucheka RM, Peltier GC, Cap AP, Bynum JA. The effects of cell type and culture condition on the procoagulant activity of human mesenchymal stromal cell-derived extracellular vesicles. J Trauma Acute Care Surg 2019;87:S74-82

[4] Chen G, Wu D, Guo W, Cao Y, Huang D, Wang H, Wang T, Zhang X, Chen H, Yu H, et al. Clinical and immunological features of severe and moderate coronavirus disease 2019. J Clin Invest 2020;130.

[5] Chen TS, Arslan F, Yin Y, Tan SS, Lai RC, Choo AB, Padmanabhan J, Lee CN, de Kleijn DP, Lim SK. Enabling a robust scalable manufacturing process for therapeutic exosomes through oncogenic immortalization of human ESC-derived MSCs. J Transl Med 2011;9:47

[6] Diao B, Wang C, Tan Y, Chen X, Liu Y, Ning L, Chen L, Li M, Liu Y, Wang G, et al Reduction and functional exhaustion of $\mathrm{T}$ cells in patients with coronavirus disease 2019 (COVID-19). Front Immunol 2020;11:827.

[7] Doeppner TR, Herz J, Gorgens A, Schlechter J, Ludwig AK, Radtke S, de Miroschedji K, Horn PA, Giebel B, Hermann DM. Extracellular vesicles improve post-stroke neuroregeneration and prevent postischemic immunosuppression. Stem Cells Transl Med 2015;4:1131-43.

\footnotetext{
${ }^{1}$ http://www.gismonline.it/images/filepdf/20200316_PostionSTEMNET_Covid19-MSCs.pdf

2 Organisation WH. Guidance for managing ethical issues in infectious disease outbreaks. World Heal Organ 2016:62.

3 FDA Guidance on Conduct of Clinical Trials of Medical Products during COVID-19 Pandemic Guidance for Industry, Investigators, and Institutional Review Boards. 2020.

${ }^{4}$ Coronavirus Treatment Acceleration Program (CTAP) | FDA n.d. https://www.fda.gov/drugs/coronavirus-covid-19-drugs/coronavirus-treatment-acceleration-program-ctap (accessed April 1, 2020).

${ }^{5}$ COVID-19 EMA pandemic Task Force (COVID-ETF): "to help EU Member States and the European Commission to take quick and coordinated regulatory action on the develop-

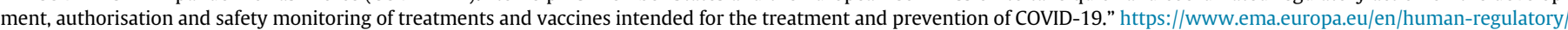
overview/public-health-threats/coronavirus-disease-covid-19/emas-governance-during-covid-19-pandemic\#covid-19-ema-pandemic-task-force-section

${ }^{6}$ https://www.ema.europa.eu/en/human-regulatory/overview/public-health-threats/coronavirus-disease-covid-19/guidance-medicine-developers-companies-covid-19

7 https://ec.europa.eu/health/sites/health/files/files/eudralex/vol-10/guidanceclinicaltrials_covid19_en.pdf

8 https://www.gov.uk/guidance/mhra-regulatory-flexibilities-resulting-from-coronavirus-covid-19\#clinical-trials
} 
[8] Gimona M, Pachler K, Laner-Plamberger S, Schallmoser K, Rohde E. Manufacturing of human extracellular vesicle-based therapeutics for clinical use. Int J Mol Sci 2017;18

[9] Hao Q Gudapati V, Monsel A, Park JH, Hu S, Kato H, Lee JH, Zhou L, He H, Lee JW. Mesenchymal stem cell-derived extracellular vesicles decrease lung injury in mice. J Immunol 2019;203:1961-72.

[10] Huang R, Qin C, Wang J, Hu Y, Zheng G, Qiu G, Ge M, Tao H, Shu Q, Xu J. Differential effects of extracellular vesicles from aging and young mesenchymal stem cells in acute lung injury. Aging (Albany NY) 2019;11:7996-8014.

[11] Khatri M, Richardson LA, Meulia T. Mesenchymal stem cell-derived extracellular vesicles attenuate influenza virus-induced acute lung injury in a pig model. Stem Cell Res Ther 2018;9:17.

[12] Khoury M, Cuenca J, Cruz FF, Figueroa FE, Rocco PRM, Weiss DJ. Current status of cell-based therapies for respiratory virus infections: applicability to COVID-19. Eur Respir J 2020:2000858

[13] Khoury M, Rocco PRM, Phinney DG, Krampera M, Martin I, Viswanathan S, Nolta JA, LeBlanc K, Galipeau J, Weiss DJ. Cell-based therapies for COVID-19: proper clinical investigations are essential. Cytotherapy 2020.

[14] Kordelas L, Rebmann V, Ludwig AK, Radtke S, Ruesing J, Doeppner TR, Epple M, Horn PA, Beelen DW, Giebel B. MSC-derived exosomes: a novel tool to treat therapy-refractory graft-versus-host disease. Leukemia 2014;28:970-3.

[15] Laffey JG, Matthay MA. Fifty Years of Research in ARDS. Cell-based therapy for acute respiratory distress syndrome. Biology and potential therapeutic value. Am J Respir Crit Care Med 2017;196:266-73.

[16] Lener T, Gimona M, Aigner L, Borger V, Buzas E, Camussi G, Chaput N, Chatterjee D, Court FA, Del Portillo HA, et al. Applying extracellular vesicles based therapeutics in clinical trials-an ISEV position paper. J Extracell Vesicles 2015;4:30087.

[17] Liu J, Chen T, Lei P, Tang X, Huang P. Exosomes released by bone marrow mesenchymal stem cells attenuate lung injury induced by intestinal ischemia reperfusion via the TLR4/NF-kappaB pathway. Int J Med Sci 2019;16:1238-44

[18] Magro C, Mulvey JJ, Berlin D, Nuovo G, Salvatore S, Harp J, Baxter-Stoltzfus A, Laurence J. Complement associated microvascular injury and thrombosis in the pathogenesis of severe COVID-19 infection: a report of five cases. Transl Res 2020.

[19] Mahida RY, Matsumoto S, Matthay MA. Extracellular vesicles: a new frontier for research in acute respiratory distress syndrome. Am J Respir Cell Mol Biol 2020.

[20] Mansouri N, Willis GR, Fernandez-Gonzalez A, Reis M, Nassiri S, Mitsialis SA Kourembanas S. Mesenchymal stromal cell exosomes prevent and revert experimental pulmonary fibrosis through modulation of monocyte phenotypes. JCI Insight 2019;4

[21] Matthay MA, Calfee CS, Zhuo H, Thompson BT, Wilson JG, Levitt JE, Rogers AJ, Gotts JE, Wiener-Kronish JP, Bajwa EK, et al. Treatment with allogeneic mesenchymal stromal cells for moderate to severe acute respiratory distress syndrome (START study): a randomised phase 2a safety trial. Lancet Respir Med 2019;7:154-62.

[22] Mehta P, McAuley DF, Brown M, Sanchez E, Tattersall RS, Manson JJ. HIh Across Speciality Collaboration, U.K. COVID-19: consider cytokine storm syndromes and immunosuppression. Lancet 2020;395:1033-4

[23] Monsel A, Zhu YG, Gennai S, Hao Q Hu S, Rouby JJ, Rosenzwajg M, Matthay MA, Lee JW. Therapeutic effects of human mesenchymal stem cell-derived microvesicles in severe pneumonia in mice. Am J Respir Crit Care Med 2015;192:324-36.

[24] Morrison TJ, Jackson MV, Cunningham EK, Kissenpfennig A, McAuley DF, O'Kane CM, Krasnodembskaya AD. Mesenchymal stromal cells modulate macrophages in clinically relevant lung injury models by extracellular vesicle mitochondrial transfer. Am J Respir Crit Care Med 2017;196:1275-86

[25] Nassar W, El-Ansary M, Sabry D, Mostafa MA, Fayad T, Kotb E, Temraz M, Saad AN, Essa W, Adel H. Umbilical cord mesenchymal stem cells derived extracellula vesicles can safely ameliorate the progression of chronic kidney diseases. Biomater Res 2016;20:21.

[26] Pachler K, Lener T, Streif D, Dunai ZA, Desgeorges A, Feichtner M, Oller M, Schallmoser K, Rohde E, Gimona M. A Good Manufacturing Practice-grade standard protocol for exclusively human mesenchymal stromal cell-derived extracellular vesicles. Cytotherapy 2017;19:458-72.

[27] Park KS, Svennerholm K, Shelke GV, Bandeira E, Lasser C, Jang SC, Chandode R, Gribonika I, Lotvall J. Mesenchymal stromal cell-derived nanovesicles ameliorate bacterial outer membrane vesicle-induced sepsis via IL-10. Stem Cell Res Ther 2019;10:231.

[28] Phinney DG. Functional heterogeneity of mesenchymal stem cells: implications for cell therapy. J Cell Biochem 2012;113:2806-12.

[29] Phinney DG, Kopen G, Righter W, Webster S, Tremain N, Prockop DJ. Donor variation in the growth properties and osteogenic potential of human marrow stromal cells. J Cell Biochem 1999;75:424-36.

[30] Qin C, Zhou L, Hu Z, Zhang S, Yang S, Tao Y, Xie C, Ma K, Shang K, Wang W, et al. Dysregulation of immune response in patients with COVID-19 in Wuhan, China. Clin Infect Dis 2020.

[31] Radtke S, Gorgens A, Liu B, Horn PA, Giebel B. Human mesenchymal and murine stromal cells support human lympho-myeloid progenitor expansion but not maintenance of multipotent haematopoietic stem and progenitor cells. Cell Cycle 2016:15:540-5.

[32] Reiner AT, Witwer KW, van Balkom BWM, de Beer J, Brodie C, Corteling RL, Gabrielsson S, Gimona M, Ibrahim AG, de Kleijn D, et al. Concise review: developing best-practice models for the therapeutic use of extracellular vesicles. Stem Cells Transl Med 2017;6:1730-9.

[33] Rohde E, Pachler K, Gimona M. Manufacturing and characterization of extracellular vesicles from umbilical cord-derived mesenchymal stromal cells for clinical testing. Cytotherapy 2019;21:581-92.

[34] Silachev DN, Goryunov KV, Shpilyuk MA, Beznoschenko OS, Morozova NY, Kraevaya EE, Popkov VA, Pevzner IB, Zorova LD, Evtushenko EA, et al. Effect of MSCs and MSCderived extracellular vesicles on human blood coagulation. Cells 2019;8.

[35] Varkouhi AK, Jerkic M, Ormesher L, Gagnon S, Goyal S, Rabani R, Masterson C, Spring C, Chen PZ, Gu FX, et al. Extracellular vesicles from interferon-gammaprimed human umbilical cord mesenchymal stromal cells reduce Escherichia coliinduced acute lung injury in rats. Anesthesiology 2019;130:778-90.

[36] Vogel W, Grunebach F, Messam CA, Kanz L, Brugger W, Buhring HJ. Heterogeneity among human bone marrow-derived mesenchymal stem cells and neural progenitor cells. Haematologica 2003;88:126-33.

[37] Wang C, Börger V, Sardari M, Murke F, Skuljec J, Pul R, Hagemann N, Dzyubenko E, Dittrich R, Gregorius J, et al. Mesenchymal stromal cell-derived small extracellular vesicles induce ischemic neuroprotection by modulating leukocytes and specifically neutrophils. Stroke 2020. STROKEAHA119028012.

[38] Willis GR, Fernandez-Gonzalez A, Anastas J, Vitali SH, Liu X, Ericsson M, Kwong A, Mitsialis SA, Kourembanas S. Mesenchymal stromal cell exosomes ameliorate experimental bronchopulmonary dysplasia and restore lung function through macrophage immunomodulation. Am J Respir Crit Care Med 2018;197:104-16.

[39] Witwer KW, Van Balkom BWM, Bruno S, Choo A, Dominici M, Gimona M, Hill AF, De Kleijn D, Koh M, Lai RC, et al. Defining mesenchymal stromal cell (MSC)derived small extracellular vesicles for therapeutic applications. J Extracell Vesicles 2019;8:1609206.

[40] Worthington EN, Hagood JS. Therapeutic use of extracellular vesicles for acute and chronic lung disease. Int J Mol Sci 2020;21:2318.

[41] Yang Y, Shen C, Li J, Yuan J, Yang M, Wang F, Li G, Li Y, Xing L, Peng L, et al. Exuberant elevation of IP-10, MCP-3 and IL-1ra during SARS-CoV-2 infection is associated with disease severity and fatal outcome. medRxiv 2020. 2020.2003.2002.20029975.

[42] Zhu YG, Feng XM, Abbott J, Fang XH, Hao Q, Monsel A, Qu JM, Matthay MA, Lee JW. Human mesenchymal stem cell microvesicles for treatment of Escherichia coli endotoxin-induced acute lung injury in mice. Stem Cells 2014;32:116-25

[43] Giebel B, Hermann DM. Identification of the right cell sources for the production of therapeutically active extracellular vesicles in ischemic stroke. Ann Transl Med 2019;7:188.

[44] Zhang B, Yeo RWY, Lai RC, Sim EWK, Chin KC, Lim SK. Mesenchymal stromal cell exosome-enhanced regulatory T-cell production through an antigen-presenting cell-mediated pathway. Cytotherapy 2018;20:687-96.

[45] Zhang B, Yin Y, Lai RC, Tan SS, Choo ABH, Lim SK. Mesenchymal Stem Cells Secrete Immunologically Active Exosomes. Stem cells and development 2014;23:1233- 